\title{
Information about The use of Drug
}

T/Lt. Surya Raj Sharma

Pharmacist

Shree Birendra Hospital

\section{Introduction}

A Drug is defined as an agent intend for use in the diagnosis, mitigation, treatment cure or prevention of disease in man or in other animals. All the drugs are chemical components, the action depends on the proper use. So various types of informations are required for our own safety health and well being. Because of the food habit most of the people are suffering from hyper acidity. So General information about Antacids are important.

\section{Antacids:}

Taken orally to relieve heartburn, sour stomach or acid indigestion. They work by neutralizing excest stomach acid. Some antacids combination also contain simethcone, Which may relieve the symptoms of excess gass. Antacids alene or in combination with simethi cone may also be used to theat the symptom of stomach or duodenai Wcers.

Antacids is the combination of magnesium having laxative effect and aluminint having the cathertic effect.

\section{Before using this Medicine}

If you are taking this medicine without a prescription carefully read and follow any precautions on the lable. For antacids the following should be considered.

\section{Allergies:}

Tell your health care professional if you have ever had any allergic reaction.

\section{Diet:}

Tell if you are on a low-sodium dielsome antacids contain large amounts of sodium.

\section{Pregnancy:}

Studies on effects in pregnancy have not been done in either humans or animals. However prolong use of high dose antacid may affect the fealus. Also sodium contaning medicines should be avoids if you tend to retain body water.

\section{Breast-feeding:}

Some aluminum, calcium or magnesium-containing antacids may pass in to breast milk, however these medicines have not been reported to cause problems in nursing babies.

\section{Children:}

Since children cannot usually describe their sypmture very well, a doctor should first check the child, precaution should be taken for premature or very young child for those who have kidney problems.

\section{Older adults:}

Aluminum-containly antacids should not be used by elderly persons with bone problems. The aluminum may cause their condition to get worse.

\section{Other Medicines:}

Although certain medicines should not be used together at in other cases different medicines may be used together even if on interaction might occur. When you are taking antacids, it is especially important that your health care professionals know if you are taking any of the following :

- Fluroquinolones: (Ciprofloxacin, Norfloxacin, Ofloxacin and Acrosoxacin) :- Antacid may decrease the effects of these medicines.

- Isoniagid taken by mouth : Aluminum, containing antacids may decrease the effects by decreasing the absorption. Isoniagid should be taken at least 1 hour before or after the antacid.

- Ketoconazoic (eg. Nizoral) or Methenamine : Antacids may decrease the effects or Ketoconazoic or Methenamine : These medicines should be taken 3 hours before the antacid.

- Tetracycline- Antacids may decrease the absorption of tetracyclines, antacids should not be taken within 3 to 4 hours of tetracyclines.

\section{Other Medical Problems:}

The presence of other medicines problems may effect the use of antacids make sure you tell your Doctor if you have any other modical problems, especially. 
Alzheimers disease (for Aluminum-containing
antacid only) or
- $\quad$ Appendicitis
- Cone Fractures
- Colitis
- $\quad$ Intestinal Blockage
- Intestinal or rectal bleeding-Antacids may
- make these condition worse
- Inflamed bowelom (Severe and Continuing)
the body to retain water and electrolytes such
as sodium and or potassium.

- Diarrhoea (Continuing) Aluminum containing antacids may cause the body to lose too much phosphorus, magnesium- Containing antacids may make diarrhoea worse.

- Edema (swelling of feet or lower legs)

- Heart disease

- Liver disease

- Toxemia of pregnancy- Use of sodium containly antacids may cause the body to retain water.

- Kidney disease- Antacids may cause higher blood levels of alumunium, calcium or magnesium which may increase the risk of serious side effects.

- Sarcoidosis Use of calcium containing antacids may cause kidney problems or too much calcium in the blood.

- Underactive- Parathyroid glands- Use with calcium containing antacids may cause too much calcium in the blood.

\section{Proper use this medicine:}

For patients taking the chewable tablets of this medicine

- Chew - the tablet well before swallowing. This is to allow the medicine to work faster and be more effective. This tablet do not have disintegrating agent.

For patients taking this medicine for a stomach or duodenal ulcer

- Take it exactly as directed and for the full time of treatment as directed by doctor to obtain maximum relief of your symptoms.

- Take it 1 \& 3 hours after means and at bedtime for best result unless otherwise specifies.
For patients taking alumunium carbonet. alumunium hydroxide to prevent kidney ats

- Drink plenty of fluids for best result un otherwise directed by Doctor.

For patients taking alumunium carbona. alumunium hydroxide for hyperphosphate

- Your Doctor may want you to follow a phosphate diet. If you have any questions at this check with your Doctor.

Dosing: The dose of an antacid will be diffece for different patients. Follow our Doctor's or for the directions on the table.

Miss Dose : If your Doctor has told you to this medicines on a regular schedule and mis dose, take it as soon as possible. However if i almost time for your next dose skip the missed and go back to your regular dosing schedule, not double doses.

Storage : To store this medicine

- Keep out of the reach of children

- Store away from heat and direct lights

- Do not store the capsule, table or Lozenge fs of this medicine in the bathroom. Near kitchen sink or in other damp places. Hea moistures may causes the medicine to bre down

- Do not keep outdate medicine or medicine: longer needed. Be sure that any discard medicine is out of the reach of children

Precaution while using this medicines:

If the doctor prescribe large doses for long timet doctor should check the progress of the patient. II is to make sure the medicine does not car. unwanted effects.

\section{Do not take this medicine:}

- If you have any signs of appendicitis: inflamed bowel (such as stomach or lat abdominal pain, cramping bloating sorex nausea or vomiting) instead check with $y$ doctor as soon as possible.

- Within 1 to 2 hours or more of taking of medicine by mouth. To do so may keep! other medicine from working properly.

For patients on a sodium-restricted diet

- Sodium bicarbonate containing antacids high sodium contains. Eoc patient takins medicine for increased stomach acid. 
Do not take it for more than 2 weeks unless otherwise directed by doctor. Antacid should be use only for occasional relief.

- If your stomach problem not helped by the antacid or if it keeps coming back. Check with your doctor.

- Using magnesives or sodium bicarbonateContaining antacids too often, or it high doses may produce a laxative effect. This happens fairly often and depends on the individuals sensitivities to the medicine

For patient taking calcium or sodium bicarbonatecontaining antacids

\section{Side effects of this medicine}

Along with its needed effects, a medicine may cause some unwanted effects. Although the following side effects occur very rarely when this medicine is taken as recommended, the may be more likely to occur if:

- Too much medicine is taken

- It is taken large dose

- It is taken for a long time

- It is taken by patients with kidney disease

Check with your doctor as soon as possible if any of the following side effects (which may be signs or overdose) occur.

For alumunium- Containing antacids (including magaldrate).

Bone Pain: Constipation (sever and continuing continuing) feeling of discomfort (continuing) loss of appetite (continuing); mood and mental change, muscle weakness; Swelling of writs or ankles; weight loss (unusual)

\section{For calcium- containing antacids:}

Constipation (severe and continuing) difficult or painful urination; frequent urge to urinate; headache (continuing); loss of appetite (continuing); mood or mental changes; muscle pain or twitching; nausea or vomiting: nervousness or restlessness; slow breathing; unpleasant taste; unusual tiredness or weakness.

\section{Side Effects of This Medicine}

Along with its needed effects, a medicine may cause some unwanted effects. Although the following side effects occur very rarely when this medicine is taken as recommended, they any be more likely to occur if- too much medicine is taken.

- $\quad$ it is taken in large dose.

- $\quad$ it is taken for a long time.

- $\quad$ it is taken by patients with kidney disease.

Check with your doctor as soon as possible if any of the following side effects (which may be signs or overdose) occur.

For aluminum-containing antacids (including magaldrate).

Bone Pain : Constipation (server and continuing) feeling of discomform (continuing); loss of appetite (continuing); mood and mental change, muscle weakness; swelling of wrists of ankles; weight loss (unusual)

\section{For Calcium-containing antacids}

Constipation (servere and continuing), difficult or painful urination; frequents urge to urinate; headache (continuing), loss of appetite (continuing) ; mood or mantes changes; muscle pain or twitching; nausea or vomiting; nervousness or restlessness; slow breathing; unpleasant taste; unusual tiredness or weakness.

For magnesium-containing antacids (including magaaldrate)

Difficult or painful urination (with magnesium triplicate); dizziness or light neatness; feeling of discomfort (continuing); irregular heartbeat; loss of appetite (continuing) mood or mental change. muscle weakness; unusual tiredness or weakness weight loss (unusual)

\section{For sodium bicarbonate-containing antacids:}

Frequent urge to urinate, headache (continuing); lots of appetite (continuing); mood or mental changes; muscle pain, mausea or vomiting; nervousness or restlessness; slow breathing; swelling of feet or lower legs; unpleasant taste; unusual tiredness or weakness.

Other side effects not mention have may also occur in some patients. If any side effects seen consult your doctor.

\section{Conclusion :}

Although the aluminium \& magnesium containing antacids are non systemic antacids have the side effects, but less than that of systemic. The benefit of antacids and other drugs depends upon the proper use. So it is adviced to take drug information from your health care professionals. 